\title{
Imidafenacin, a novel antimuscarinic agent, is alternative to antimuscarinic agents in patients with overactive bladder syndrome:An updated systematic review and Meta-analysis of randomized controlled trials
}

\section{Jia-Pei Wu}

Sichuan University West China Hospital

\section{Liao Peng}

Sichuan University West China Hospital

\section{Xiao Zeng}

Sichuan University West China Hospital

$\mathrm{HaO} \mathrm{Li}$

Sichuan University West China Hospital

\section{Hong Shen}

Sichuan University West China Hospital

De-yi Luo (D luodeyi1985@163.com )

Sichuan University West China Hospital https://orcid.org/0000-0002-7009-9398

\section{Research article}

Keywords: Imidafenacin; Novel antimuscarinic agent; Antimuscarinics; Anticholinergic drug; Overactive bladder syndrome; Quality of life; Meta-analysis

Posted Date: July 24th, 2019

DOl: https://doi.org/10.21203/rs.2.11855/v1

License: (9) (1) This work is licensed under a Creative Commons Attribution 4.0 International License. Read Full License 


\section{Abstract}

Purpose Previous study included limited number of randomized controlled trials (RCTs) and compared limited parameters post the treatment of imidafenacin and other anticholinergic drugs (ADs) for overactive bladder syndrome (OAB) and controversy about the superiority of those ADs still remains. We aim to update the evidence and provide better clinical guidance. Methods $A$ systematic search in PubMed, Embase, ClinicalTrial.gov, and Cochrane Library Central Register of Controlled Trials was conducted from January 2007 to April 2019. Meta-analysis of all published RCTs comparing imidafenacin with other ADs in patients with OAB was performed. The primary outcomes were the changes in OAB symptoms and OAB Symptom Score (OABSS). Secondary outcomes included adverse events (AEs) and dropout rate related to AEs. Results A total of 6 studies including 7 RCTs involving 1,430 patients with mean follow-up of 23.43 weeks were included. All ADs improved OAB symptoms. In regard to efficacy, those drugs had similar efficacy in voids, urgency episodes, urgency incontinence episodes, incontinence episodes and in OABSS. However, Imidafenacin had a better performance in the reduction of nocturia episodes ( $M D=-0.24,95 \% \mathrm{Cl}-0.44$ to- $0.04, \mathrm{P}=0.02)$. Moreover, Imidafenacin was associated with statistically lower dry mouth rate $(R R=0.87,95 \% \mathrm{Cl} 0.75-1.00, P=0.04)$, lower constipation rate $(R R=0.68,95 \% \mathrm{Cl} 0.50$ $0.93, \mathrm{P}=0.01)$ and lower $\mathrm{AE}-$ related withdrawal rate $(\mathrm{RR}=0.51,95 \% \mathrm{Cl} 0.29-0.89, \mathrm{P}=0.02)$. There was no significant difference in terms of other complications. Conclusions In conclusion, Imidafenacin was comparable with other ADs in the treatment of OAB. Moreover, imidafenacin presented lower dry mouth rate, lower constipation rate and higher adherence and persistence.

\section{Introduction}

Overactive bladder syndrome (OAB), usually accompanied by frequency and nocturia, is characterized by urinary urgency, with or without urgency urinary incontinence[1,2]. Etiology and pathologic mechanism remain unclear. $O A B$ is a chronic and debilitating condition, whose overall prevalence is estimated $11.8 \%-16.9 \%$ in US[3,4]. OAB symptoms can usually affect work productivity, social relationships, and sleep patterns, causing anxiety and depression, and lead to decline of life quality[5].

Oral antimuscarinic agents (i.e., solifenacin, propiverine, darifenacin, and fesoterodine) have been the mainstay of pharmacotherapy for the treatment of OAB[6]. However, these antimuscarinics lack bladder selectivity and result in dry mouth, constipation, and other adverse events, decreasing patient satisfaction and leading to discontinuation or switching[7,8].

Imidafenacin, which is a novel antimuscarinic agent with high bladder selectivity, was used to treat OAB in Japan since 2007 and had higher affinity for the M1 and M3 muscarinic receptor subtypes and lower affinity for the M2 subtype[9,10]. Study had confirmed the efficacy and safety of imidafenacin compared with placebo [11]. However, whether it is a better choice for OAB patients compared with other anticholinergic drugs still remains unclear.

Huang et al.[12] analyzed only 4 randomized controlled trials (RCTs) in 2015, and captured limited parameters to compare the efficacy (without nocturia episodes) and safety (only dry mouth and constipation rate) of those antimuscarinic agents and controversy about the superiority of those antimuscarinics still remains.

Hence, this present study aims to include more relevant RCTs and analyze more parameters to compare the efficacy and safety of imidafenacin and other antimuscarinics for $\mathrm{OAB}$, and provide updated evidence for clinical practice.

\section{Materials And Methods}

\subsection{Search strategy}

The current study was performed according to the Preferred Reporting Items of Systematic Reviews and Meta-Analyses guideline[13]. A systematic search in PubMed, Embase, ClinicalTrial.gov, and Cochrane Library Central Register of Controlled Trials was conducted from January 2007 to April 2019. The electronic search was performed to capture all relevant studies using the following search strategy: (Overactive bladder OR OAB) AND (Novel antimuscarinic agent OR Antimuscarinics OR Cholinergic drug OR Imidafenacin OR tolterodine OR darifenacin OR solifenacin OR fesoterodine OR propiverine OR botulinum toxin) AND Quality of life. The procedure of retrieving and evaluating the articles was conducted independently by two reviewers (JP-W and LP), and differences were solved through a discussion in group. The selection procedure was presented in Fig 1 . This study did not need IRB approval because the data were directly obtained from studies freely available on the internet instead of being collected from institutions or patients. 


\subsection{Study selection and exclusion criteria}

The Eligible for inclusion: (1) published RCTs compared imidafenacin with at least one anticholinergic drug (AD) in patients with $O A B ;(2)$ studies provided main outcome data evaluating the safety and tolerability of the these antimuscarinics.

The exclusion criteria: (1) descriptive studies without comparison between imidafenacin with other ADs; (2) a type of review, metaanalysis, letter, case report, or studies based on animals or on children; (3) duplications and repeated analyses. There were no exclusion criteria based on publication status or language.

\subsection{Outcomes}

The primary outcomes were used to evaluate the effectiveness including overactive bladder symptom score (OABSS)[14], and the change in $O A B$ symptoms (urgency episode/day, nocturia episode/day, urgency incontinence episode/day, incontinence episode/day, urine volume voided per micturition, and micturition/day). Secondary outcomes were adverse events (AEs), such as dry mouth, constipation, prolongation of intervals of electrocardiogram (ECG), blurred version, headache, stomach discomfort, nausea, dry eyes, postvoid residual volume (PVR), urinary tract infection (UTI), abdominal pain, and dropout rate related to AEs.

\subsection{Assessment for risk of bias}

The risk of bias (ROB) across RCTs was assessed using a ROB graph generated through Review Manager software according to Cochrane hand book. ROB domains were judged as low, high, or unclear risk.

\subsection{Statistical analysis}

Review Manager 5.3 (Cochrane Collaboration, Oxford, UK) was used to perform all analyses. Mean difference (MD) and relative risk (RR) with 95\% confidential interval (Cl) were calculated for continuous and dichotomous outcomes, respectively. Heterogeneity was evaluated by $I^{2}$ test, with significance set at $P<0.05$. $I^{2}$ values of 25,50 , and $75 \%$ corresponded to low, medium, and high levels of heterogeneity, respectively. The fixed-effect method was used for studies without significant heterogeneity $\left(I^{2}\right.$ values $\left.<50 \%\right)$, and random-effect method was used with $\mathrm{I}^{2}$ values $\geq 50 \%$. Sensitivity analyses were performed If a huge heterogeneity was detected. Publication bias was calculated using funnel plot in Review Manager.

\section{Results}

\subsection{Literature search and study characteristics}

Totally, 138 records have been searched using the items in searching strategies from January 2007 to May 2019. Duplications $(n=20)$ and non-comparative articles $(n=53)$ were excluded, respectively. Then, 65 full-text papers were assessed independently by two reviewers ( $L P$ and $X Z$ ) for eligible according to previous inclusion criteria. Finally, 6 studies published in English including 7 RCTs[15-20] with mean follow-up of 23.43 (2-52) weeks were captured and included in our meta-analysis. According to the study design in LIST study[17], there were two RCTs in this study regarding long-term and short-term follow-up, with different baseline and endpoints. 7 RCTs included very overall outcome data regarding both efficacy and safety of those ADs. Moreover, the ROB across included studies was relatively low as depicted in Fig 2. Characteristics of included RTCs were presented in Table 1.

\subsection{Efficiency}

\subsubsection{Urgency episodes per day}

Considering that $O A B$ was characterized by urinary urgency, the change of urgency episodes/day was a very important indicator for the improvement of OAB symptom. As expected, imidafenacin compared with other ADs had no statistically difference in 4,8,12-week urgency episodes ( 4 weeks: $\mathrm{MD}=0.31,95 \% \mathrm{Cl}-0.22$ to $0.83, \mathrm{P}=0.25 ; 8$ weeks: $\mathrm{MD}=-0.10,95 \% \mathrm{Cl}-0.63$ to $0.43, \mathrm{P}=0.70 ; 12$ weeks: $\mathrm{MD}=0.21,95 \% \mathrm{Cl}-0.14$ to $0.56, \mathrm{P}=0.24)$. Interestingly, other $\mathrm{ADs}$ presented more reduction in urgency episodes in 2 weeks ( $\mathrm{MD}=0.9$, $95 \% \mathrm{Cl} 0.17-1.63, \mathrm{P}=0.02$ ), which possibly implied that imidafenacin took longer ( $>2$ weeks) to show the best effects (Fig 3 ).

\subsubsection{Urgency incontinence episodes per day}


Subgroup analysis of different follow-up periods regarding urgency incontinence episodes in 4 RCTs was performed. No statistically difference was observed ( 2 weeks: $\mathrm{MD}=-0.20,95 \% \mathrm{Cl}-0.66$ to $0.26, \mathrm{P}=0.40 ; 4$ weeks: $\mathrm{MD}=-0.03,95 \% \mathrm{Cl}-0.37$ to $0.32, \mathrm{P}=0.89 ; 8$ weeks: $\mathrm{MD}=-0.17,95 \% \mathrm{Cl}-0.51$ to $0.18, \mathrm{P}=0.34: 12$ weeks: $\mathrm{MD}=-0.01,95 \% \mathrm{Cl}-0.23$ to $0.20, \mathrm{P}=0.89$ ) (Fig 4).

\subsubsection{Micturition per day and urine volume voided per micturition}

Similarly, subgroup analysis of 2,4,8,12-week micturition showed no statistically difference between imidafenacin and other antimuscarinics ( 2 weeks: $\mathrm{MD}=-0.3,95 \% \mathrm{Cl}-0.96$ to $0.36, \mathrm{P}=0.38 ; 4$ weeks: $\mathrm{MD}=0.12,95 \% \mathrm{Cl}-0.10$ to $0.34, \mathrm{P}=0.27 ; 8$ weeks: $\mathrm{MD}=0.23$, $95 \% \mathrm{Cl} 0.00$ to $0.47, \mathrm{P}=0.05 ; 12$ weeks: $\mathrm{MD}=0.18,95 \% \mathrm{Cl}-0.07$ to $0.42, \mathrm{P}=0.15$ ) (Fig 5). As expected, imidafenacin had similar performance in mean urine volume voided per micturition ( $\mathrm{MD}=-5.38,95 \% \mathrm{Cl}-10.83$ to $0.07, \mathrm{P}=0.05)$ (Fig 6).

\subsubsection{Nocturia episodes}

Gratifyingly, imidafenacin had better performance in terms of nocturia episodes ( $\mathrm{MD}=-0.24,95 \% \mathrm{Cl}-0.44$ to $-0.04, \mathrm{P}=0.02)(\mathrm{Fig} 7)$, indicating that imidafenacin could reduce the number of nighttime voids, increase bladder capacity and improve sleep disorders.

\subsubsection{Incontinence episodes per day}

In line with urgency incontinence episodes/ day, imidafenacin had similar performance in reduction of incontinence episodes (2 weeks: $\mathrm{MD}=0.05,95 \% \mathrm{Cl}-0.87$ to $0.97, \mathrm{P}=1.00 ; 4$ weeks: $\mathrm{MD}=0.36,95 \% \mathrm{Cl}-0.34$ to $1.06, \mathrm{P}=0.31 ; 12$ weeks: $\mathrm{MD}=0.10,95 \% \mathrm{Cl}-0.18$ to $0.37, \mathrm{P}=0.48)$. (Fig 8)

\subsubsection{OABSS}

OABSS, integrating four symptoms into a single score, was a useful tool to evaluate the improvement of OAB symptoms and life quality[14].

In the current study, OABSS was reported in 4 RCTs involving 465 patients (Fig 9). There was no statistically differences between imidafenacin and other ADs throughout the administration period ranging from 2-52 week (2 weeks: $\mathrm{MD}=0.20,95 \% \mathrm{Cl}-1.46$ to 1.86 , $\mathrm{P}=0.81$; 4 weeks: $\mathrm{MD}=0.39,95 \% \mathrm{Cl}-0.35$ to $1.12, \mathrm{P}=0.30 ; 8$ weeks: $\mathrm{MD}=0.80,95 \% \mathrm{Cl}-0.89$ to $2.49, \mathrm{P}=0.35 ; 12$ weeks: $\mathrm{MD}=0.48,95 \% \mathrm{Cl}$ -0.27 to $1.24, \mathrm{P}=0.21 ; 24$ weeks: $\mathrm{MD}=0.86,95 \% \mathrm{Cl}-0.23$ to $1.95, \mathrm{P}=0.12 ; 52$ weeks: $\mathrm{MD}=-0.28,95 \% \mathrm{Cl}-1.24$ to $0.68, \mathrm{P}=0.56$ ). The overall findings were accordant with data in urgency, urgency incontinence, voids, and urine volume voided per micturition, suggesting that imidafenacin compared with other ADs had similar efficacy for the improvements of OAB symptoms. However, imidafenacin could significantly reduce the nocturia episodes. Cheerfully, no publication bias was identified (Fig 10).

\subsection{Safety}

As reported, dry mouth was the most common adverse event in the course of anticholinergic drug therapy[6,21]. Gratifyingly, the current study reported imidafenacin had a significantly lower risk in dry mouth (RR=0.87, 95\% $\mathrm{Cl} 0.75$ to 1.00, $P=0.04)$ (Fig 11), significantly lower constipation rate $(\mathrm{RR}=0.68,95 \% \mathrm{Cl} 0.50$ to $0.93, \mathrm{P}=0.01)$ (Fig 11), and significantly lower dropout rate related to AEs (RR=0.55, 95\% Cl 0.27 to $1.14, \mathrm{P}=0.02$ ) (Fig 12).

Simultaneously, imidafenacin did not show a higher risk in blurred vision, dry eyes, UTI, headache, stomach discomfort, nausea, and abdominal pain (Fig 11), as well as in PVR (4 weeks: $\mathrm{MD}=-3.60,95 \% \mathrm{Cl}-9.76$ to $2.56 \mathrm{P}=0.25: 12$ weeks: $\mathrm{MD}=2.37,95 \% \mathrm{Cl}-3.81$ to 8.55 , $\mathrm{P}=0.45$ : 24 weeks: $\mathrm{MD}=2.50,95 \% \mathrm{Cl}-7.79$ to $12.79, \mathrm{P}=0.63$ : 52 weeks: $\mathrm{MD}=-10.44,95 \% \mathrm{Cl}-22.30$ to $1.43, \mathrm{P}=0.08)$ (Fig 13).

Regarding the cardiovascular risk, no difference was reported in the prolongation of QT intervals of ECG range 4-52 weeks ( 4 weeks: $\mathrm{MD}=1.31,95 \% \mathrm{Cl}-9.12$ to $11.74, \mathrm{P}=0.81 ; 12$ weeks: $\mathrm{MD}=0.82,95 \% \mathrm{Cl}-10.07$ to $11.71, \mathrm{P}=0.88: 52$ weeks: $\mathrm{MD}=5.90,95 \% \mathrm{Cl}-11.97$ to 23.77, $\mathrm{P}=0.52)$ (Fig 14).

\section{Discussion}

\section{Principal findings}


$O A B$ is a chronic and debilitating disease. The long-term efficacy and tolerability of treatments will be very important regarding it is a QOL-related disease. Antimuscarinics (i.e., solifenacin, propiverine, and tolterodine) have been used as first-line pharmacotherapy for $\mathrm{OAB}$, however, a current study reported that discontinuation rate was $84 \%$ and switching rate was $4 \%$ in 12 -month follow-up duo to AEs[21]. Thus, it is necessary to find a novel anticholinergic drug with higher tolerability in the long-term follow-up. Imidafencin

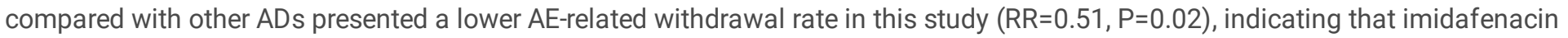
had higher tolerability and adherence in 23.43-week follow-up. $0.1 \mathrm{mg}$ twice daily or $0.2 \mathrm{mg}$ once daily was recommended clinical dosage for the treatment of $O A B$.

It is clear that imidafenacin has more efficacy than placebo[11]. However, controversy about priority of imidafenacin and other antimuscarinics still unclear although Huang et al.(published in 2015)[12] had conducted a meta-analysis previously. Researcher included only 4 articles and captured limited parameters to compare the efficacy and safety of three antimuscarinic agents (imidafenacin vs. solifenacin and propiverine). The advantage of the current meta-analysis is that we included three more highquality RCTs with totally 5 antimuscarinics and overall analyzed parameters (2-52 weeks) regarding both efficacy and safety of those drugs, especially adding overall indicators to evaluate the safety. One serious problem in previous study was authors used data from each visit rather than variation compared with baseline, and there was no comparability if the primary data were not consistent. Another advantage of present study was that heterogeneity was generally low across all analyses.

In regard to effectiveness, there was no statistically difference between imidafenacin and other antimuscarinics in 4,8,12-week urgency episodes; 2,4,8,12-week urgency incontinence episodes; 2, 4,12-week incontinence episodes; 2,4,8,12-week voids; 4,8,12-week urine volume voided per micturition, and in 2,4,8,12,24,52-week OABSS. However, imidafenacin had less nocturia episodes (MD=-0.24, $\mathrm{P}=0.02$ ), which was consistent with the hypothesis that imidafenacin could reduce the number of nighttime voids, increase bladder capacity and improve sleep disorders[22,23]. Notably, other ADs presented less 2-week urgency episodes (MD=0.9, 95\% $\mathrm{Cl}$ 0.17-1.63, $\mathrm{P}=0.02)$, despite all ADs improved this symptom. The possible explanation is that imidafenacin takes longer ( $>2$ weeks) to show the best results. Better performance in 4,8,12-week urgency episodes can nicely confirm our guess. According to pharmacokinetics and pharmacodynamics, imidafenacin distributed predominantly to the bladder and exerted more selective and longer-lasting effect on the bladder than other tissues, despite the short blood half-life[24]. Moreover, the imidafenacin excreted in urine also play an important role in pharmacokinetic and pharmacological selectivity[24].

In terms of safety, Huang et al. only reported dry mouth rate, constipation rate and total number of AEs. However, significant lower dry mouth rate and lower constipation rate were accordant with Huang's data. We believed the higher selectivity in bladder than salivary gland and digest system could explain for the results. As expected, these was no statistically difference in PVR, QT intervals, blurred version, headache, stomach discomfort, nausea, dry eyes, and abdominal pain. Blood-brain barrier blocks the binding to the brain's anticholinergic receptor[24], suggesting imidafenacin will not increase the risk for patient with cardiovascular diseases[25]. Similarly, imidafenacin bound rarely to the agents on stomach and colon resulting in less digest-system-related AEs[24]. Taken together, these findings confirmed the safety of imidafenacin.

\section{Clinical decision and limitations}

Since the prevalence of $O A B$ increases with age, elderly individuals often associated with polypharmacy might experience a higher antimuscarinics burden[26]. What is worse, nocturia, greatly under-reported by both physicians and OAB patients, was perceived as a normal part of the aging process[27]. Nocturia was associated with increased overall mortality in patients voiding at least three times nightly[28] and was an important risk factor for nighttime falls in the elderly[29]. In the present study, imidafenacin compared with other antimuscarinics had better efficacy in reduction of nocturia episodes ranging from 4-12 weeks. Nevertheless, the cause of nocturia was multifactorial, which included low bladder capacity (including OAB), nocturnal overproduction of urine (NP), and a combination of NP and low bladder capacity. A prospective study concluded that imidafenacin led to an increase in bladder capacity 
and a decrease in nocturnal urine volume[23]. Another result from a phase III RCT drew the same conclusion that imidafenacin improved both nocturia and nocturnal polyuria[22]. Nevertheless, Linda et al. reported that solifenacin could not improve nocturia symptoms in $\mathrm{OAB}$ patients with NP[30]. The findings suggested that imidafenacin may be a potential choice for elders with refractory nocturia caused by $O A B$, despite the evidence directly compared imidafenacin with other ADs on nocturia affected by OAB was lacking.

OAB syndrome often affects elderly patients who may present cardiovascular comorbidities[25]. According to our analysis, imidafenacin had no influence on the prolongation of 4,12,52-week QT intervals of ECG and all the RCTs reported few imidafenacinrelated cardiovascular events. Therefore, we recommend imidafenacin as a preferable treatment for elder patients with cardiovascular diseases for the benefits of pharmacokinetics and pharmacodynamics[24]. Of course, regular follow-ups and ECG monitoring are necessary throughout the administration period in selected populations such as patients aged $>80$ years, accompanied by coronary heart disease or congestive heart failure.

Despite a network meta-analysis[31] was performed recently, only few included articles studied the effect of imidafenacin and only voids, leaks, and dry mouth were analyzed in their study. Considering that imidafenacin was not the main body of study and important outcomes, such as OABSS, urgency episodes, nocturia episodes, and drug-related adverse events, were lacking, we included more RCTs and analyzed overall parameters in an updated meta-analysis that chiefly investigated the effects of imidafenacin to provide a better clinical guidance.

Our study should not be interpreted without limitations. First, although we have included very comprehensive evaluation parameters regarding both efficacy and safety, only imidafenacin vs. solifenacin, propiverine, tolterodine and fesoterodine were performed. Future studies should focus on the comparison between imidafenacin and other antimuscarinics, such as botulinum toxin and darifenacin. Second, the current research mainly focuses on Asian patients (Japan, Korea, and Russia). Considering that patients have their own characteristics in different countries, the researches in other regions are also needed, especially in western countries. Third, the data on nocturia were available in only one RCT ranging from 4-12 weeks, and there was no difference between two groups in the original article. However, imidafenacin had a better performance by our statistical analysis using the same data. There may be some statistic errors in the original study. Thus, to confirm the effects of imidafenacin compared with other ADs on nocturia, more high-quality long-term RCTs are needed.

\section{Conclusion}

In the present study, we founded that patients treated with imidafenacin and other antimuscarinics had similar efficacy in regard to OABSS and improvements of OAB symptoms, except imidafenacin had less nocturia episodes, suggesting that imidafenacin may be a potential choice for patients with nocturia caused by OAB. Duo to lower dry month rate, lower constipation rate and less withdrawals, imidafenacin is preferable for patients who need long-term medications. Moreover, imidafenacina, without increasing cardiovascular risk, is an optimal therapy for elder patients accompanied with cardiovascular diseases.

\section{Declarations}

Authors' Contribution: L Peng, J-P Wu and X Zeng: Data Collection, Data analysis, Manuscript writing; H Shen, H Li: Data collection and management; L Peng and D-Y Luo: Protocol/project development.

Funding: This study was funded by the National Natural Science Fund of China(Grant Nos.81770673) and 1.3.5 project for disciplines of excellence, West China Hospital, Sichuan University (Grant Nos.ZY2017310). 
Conflict of Interest: The authors declare that they have no conflict of interest.

Ethical approval: This article does not contain any studies with human participants or animals performed by any of the authors.

Informed consent: Not applicable.

\section{References}

1. Abrams P, Cardozo L, Fall M, Griffiths D, Rosier P, Ulmsten U, van Kerrebroeck P, Victor A, Wein A (2002) The standardisation of terminology of lower urinary tract function: Report from the standardisation sub-committee of the international continence society. American Journal of Obstetrics and Gynecology 187 (1):116-126. doi:10.1067/mob.2002.125704

2. Drake MJ (2014) Do we need a new definition of the overactive bladder syndrome? ICI-RS 2013. Neurourology and urodynamics 33 (5):622-624. doi:10.1002/nau.22609

3. Irwin DE, Milsom I, Hunskaar S, Reilly K, Kopp Z, Herschorn S, Coyne K, Kelleher C, Hampel C, Artibani W, Abrams P (2006) Population-based survey of urinary incontinence, overactive bladder, and other lower urinary tract symptoms in five countries: results of the EPIC study. European urology 50 (6):1306-1314; discussion 1314-1305. doi:10.1016/j.eururo.2006.09.019

4. Stewart WF, Van Rooyen JB, Cundiff GW, Abrams P, Herzog AR, Corey R, Hunt TL, Wein AJ (2003) Prevalence and burden of overactive bladder in the United States. World J Urol 20 (6):327-336. doi:10.1007/s00345-002-0301-4

5. Milsom I, Kaplan SA, Coyne KS, Sexton CC, Kopp ZS (2012) Effect of bothersome overactive bladder symptoms on health-related quality of life, anxiety, depression, and treatment seeking in the United States: results from EpiLUTS. Urology 80 (1):90-96. doi:10.1016/j.urology.2012.04.004

6. Andersson K-E (2004) Antimuscarinics for treatment of overactive bladder. The Lancet Neurology 3 (1):46-53. doi:10.1016/s1474-4422(03)00622-7

7. Chapple CR, Nazir J, Hakimi Z, Bowditch S, Fatoye F, Guelfucci F, Khemiri A, Siddiqui E, Wagg A (2017) Persistence and Adherence with Mirabegron versus Antimuscarinic Agents in Patients with Overactive Bladder: A Retrospective Observational Study in UK Clinical Practice. European urology 72 (3):389-399. doi:10.1016/j.eururo.2017.01.037

8. Akino H, Namiki M, Suzuki K, Fuse H, Kitagawa Y, Miyazawa K, Fujiuchi Y, Yokoyama O (2014) Factors influencing patient satisfaction with antimuscarinic treatment of overactive bladder syndrome: results of a real-life clinical study. International journal of urology : official journal of the Japanese Urological Association 21 (4):389-394. doi:10.1111/iju.12298

9. Kobayashi F, Yageta Y, Segawa M, Matsuzawa S (2007) Effects of imidafenacin (KRP-197/ONO-8025), a new anti-cholinergic agent, on muscarinic acetylcholine receptors. High affinities for M3 and M1 receptor subtypes and selectivity for urinary bladder over salivary gland. Arzneimittel-Forschung 57 (2):92-100. doi:10.1055/s-0031-1296589

10. Kobayashi F, Yageta Y, Yamazaki T, Wakabayashi E, Inoue M, Segawa M, Matsuzawa S (2007) Pharmacological effects of imidafenacin (KRP-197/ONO-8025), a new bladder selective anti-cholinergic agent, in rats. Comparison of effects on urinary bladder capacity and contraction, salivary secretion and performance in the Morris water maze task. Arzneimittel-Forschung 57 (3):147-154. doi:10.1055/s-0031-1296598

11. Homma Y, Yamaguchi T, Yamaguchi O (2008) A randomized, double-blind, placebo-controlled phase II dose-finding study of the novel anti-muscarinic agent imidafenacin in Japanese patients with overactive bladder. International journal of urology : official journal of the Japanese Urological Association 15 (9):809-815. doi:10.1111/j.1442-2042.2008.02104.x

12. Huang W, Zong H, Zhou X, Zhang Y (2015) Efficacy and safety of imidafenacin for overactive bladder in adult: a systematic review and meta-analysis. International urology and nephrology 47 (3):457-464. doi:10.1007/s11255-015-0916-1

13. Moher D, Liberati A, Tetzlaff J, Altman DG, Group P (2009) Preferred reporting items for systematic reviews and meta-analyses: the PRISMA statement. BMJ (Clinical research ed) 339:b2535. doi:10.1136/bmj.b2535

14. Homma Y, Yoshida M, Seki N, Yokoyama O, Kakizaki H, Gotoh M, Yamanishi T, Yamaguchi O, Takeda M, Nishizawa O (2006) Symptom assessment tool for overactive bladder syndrome-overactive bladder symptom score. Urology 68 (2):318-323. doi:10.1016/j.urology.2006.02.042

Page $7 / 20$ 
15. Homma Y, Yamaguchi O, Imidafenacin Study G (2009) A randomized, double-blind, placebo- and propiverine-controlled trial of the novel antimuscarinic agent imidafenacin in Japanese patients with overactive bladder. International journal of urology : official journal of the Japanese Urological Association 16 (5):499-506. doi:10.1111/j.1442-2042.2009.02286.x

16. Yokoyama T, Koide T, Hara R, Fukumoto K, Miyaji Y, Nagai A (2013) Long-term safety and efficacy of two different antimuscarinics, imidafenacin and solifenacin, for treatment of overactive bladder: a prospective randomized controlled study. Urologia internationalis 90 (2):161-167. doi:10.1159/000345055

17. Zaitsu M, Mikami K, Ishida N, Takeuchi T (2011) Comparative Evaluation of the Safety and Efficacy of Long-Term Use of Imidafenacin and Solifenacin in Patients with Overactive Bladder: A Prospective, Open, Randomized, Parallel-Group Trial (the LIST Study). Adv Urol 2011:854697. doi:10.1155/2011/854697

18. Park C, Park J, Choo MS, Kim JC, Lee JG, Lee JZ, Lee KS, Kim DY, Lee SJ, Seo JT (2014) A randomised, prospective double-blind, propiverine-controlled trial of imidafenacin in patients with overactive bladder. International journal of clinical practice 68 (2):188-196. doi:10.1111/ijcp.12255

19. Lee KS, Park B, Kim JH, Kim HG, Seo JT, Lee JG, Jang Y, Choo MS (2013) A randomised, double-blind, parallel design, multiinstitutional, non-inferiority phase IV trial of imidafenacin versus fesoterodine for overactive bladder. International journal of clinical practice 67 (12):1317-1326. doi:10.1111/ijcp.12272

20. Pushkar DY, Kasyan GR, Kolontarev KB, Sharvadze GG, Mukhametshina El (2019) Randomized, open-label, tolterodine-controlled, comparative study of the novel antimuscarinic agent imidafenacin in patients with overactive bladder. Neurourology and urodynamics. doi:10.1002/nau.23980

21. Sussman D, Yehoshua A, Kowalski J, Lee W, Kish J, Chaudhari S, Murray B (2017) Adherence and persistence of mirabegron and anticholinergic therapies in patients with overactive bladder: a real-world claims data analysis. International journal of clinical practice 71 (3-4). doi:10.1111/ijcp.12824

22. Yokoyama O, Homma Y, Yamaguchi $O$ (2013) Imidafenacin, an antimuscarinic agent, improves nocturia and reduces nocturnal urine volume. Urology 82 (3):515-520. doi:10.1016/j.urology.2013.05.017

23. Wada N, Watanabe M, Kita M, Matsumoto S, Osanai H, Yamaguchi S, Numata A, Fujisawa M, Saga Y, Hou K, luchi H, Niibori D, Kura T, Taniguchi A, Kunieda M, Nakata Y, Kakizaki H (2012) Effect of imidafenacin on nocturia and sleep disorder in patients with overactive bladder. Urologia internationalis 89 (2):215-221. doi:10.1159/000339750

24. Yamada S, Seki M, Ogoda M, Fukata A, Nakamura M, Ito Y (2011) Selective binding of bladder muscarinic receptors in relation to the pharmacokinetics of a novel antimuscarinic agent, imidafenacin, to treat overactive bladder. J Pharmacol Exp Ther 336 (2):365-371. doi:10.1124/jpet.110.172288

25. Rosa GM, Bauckneht M, Scala C, Tafi E, Leone Roberti Maggiore U, Ferrero S, Brunelli C (2013) Cardiovascular effects of antimuscarinic agents in overactive bladder. Expert Opin Drug Saf 12 (6):815-827. doi:10.1517/14740338.2013.813016

26. Yoshida M, Kato D, Nishimura T, Van Schyndle J, Uno S, Kimura T (2018) Anticholinergic burden in the Japanese elderly population: Use of antimuscarinic medications for overactive bladder patients. International journal of urology : official journal of the Japanese Urological Association 25 (10):855-862. doi:10.1111/iju.13758

27. Lundgren R (2004) Nocturia: a new perspective on an old symptom. Scandinavian journal of urology and nephrology 38 (2):112116. doi:10.1080/00365590310020033

28. Asplund R (1999) Mortality in the elderly in relation to nocturnal micturition. BJU international 84 (3):297-301

29. Stewart RB, Moore MT, May FE, Marks RG, Hale WE (1992) Nocturia: a risk factor for falls in the elderly. Journal of the American Geriatrics Society 40 (12):1217-1220

30. Brubaker L, FitzGerald MP (2007) Nocturnal polyuria and nocturia relief in patients treated with solifenacin for overactive bladder symptoms. International urogynecology journal and pelvic floor dysfunction 18 (7):737-741. doi:10.1007/s00192-006-0239-y

31. Herbison P, McKenzie JE (2019) Which anticholinergic is best for people with overactive bladders? A network meta-analysis. Neurourology and urodynamics 38 (2):525-534. doi:10.1002/nau.23893

\section{Figure Legends}

Fig 1 PRISMA flowchart of study selection. 
Fig 2 Results of assessment of risk of bias (ROB). ROB domains were judged as low(green), high(red), or unclear risk(yellow).

Fig 3 Forest Plot for urgency episodes per day post the treatment of imidafenacin and other antimuscarinics.

Fig 4 Forest Plot for urgency incontinence episodes per day.

Fig 5 Forest Plot for micturition per day.

Fig 6 Forest Plot for urine volume voided per micturition.

Fig 7 Forest Plot for nocturia episodes post the treatment of imidafenacin and other cholinergic drugs.

Fig 8 Forest Plot for incontinence episodes per day post the treatment of imidafenacin and controlled group.

Fig 9 Forest Plot for overactive bladder symptom score (OABSS).

Fig 10 Publication bias in overactive bladder symptom score (OABSS).

Fig 11 Forest Plot for overall adverse events post the treatment of antimuscarinics.

Fig 12 Forest Plot for dropout rate related to adverse events for the treatment of imidafenacin and other antimuscarinics.

Fig 13 Forest Plot for postvoid residual volume (PVR).

Fig 14 Forest Plot for prolongation of QT intervals of electrocardiogram (ECG).

\section{Table}


Table 1 Characteristics of included studies.

\begin{tabular}{|c|c|c|c|c|c|c|c|c|c|}
\hline References & Year & Design & Country & Groups(n) & Age & Dosage & Usage & Withdrawal* & $\begin{array}{l}\text { Follow- } \\
\text { up(weeks) }\end{array}$ \\
\hline \multirow[t]{2}{*}{ Masayoshi } & 2011 & 2RCTs & Japan & imidafenacin(21) & $70.2 \pm 6.5$ & $0.1 \mathrm{mg}$ & $\begin{array}{l}\text { twice } \\
\text { daily }\end{array}$ & 0 & 12,52 \\
\hline & & & & solifenacin(20) & $69.8 \pm 7.7$ & $5 \mathrm{mg}$ & $\begin{array}{l}\text { once } \\
\text { daily }\end{array}$ & 3 & \\
\hline \multirow[t]{2}{*}{ Teruhiko } & 2013 & $\mathrm{RCT}$ & Japan & imidafenacin(55) & $72.0 \pm 9.3$ & $0.1 \mathrm{mg}$ & $\begin{array}{l}\text { twice } \\
\text { daily }\end{array}$ & 3 & $\begin{array}{l}4,12,24 \\
52\end{array}$ \\
\hline & & & & solifenacin (54) & $70.4 \pm 13.0$ & $5 \mathrm{mg}$ & $\begin{array}{l}\text { once } \\
\text { daily }\end{array}$ & 7 & \\
\hline \multirow[t]{2}{*}{ Yukio } & 2009 & $\mathrm{RCT}$ & Japan & imidafenacin(324) & $57.7 \pm 12.7$ & $0.1 \mathrm{mg}$ & $\begin{array}{l}\text { twice } \\
\text { daily }\end{array}$ & 11 & $4,8,12$ \\
\hline & & & & propiverine(310) & $59.8 \pm 11.9$ & $20 \mathrm{mg}$ & $\begin{array}{l}\text { once } \\
\text { daily }\end{array}$ & 19 & \\
\hline \multirow[t]{2}{*}{ Park C } & 2014 & $\mathrm{RCT}$ & Korea & imidafenacin(75) & $58.31 \pm 11.45$ & $0.1 \mathrm{mg}$ & $\begin{array}{l}\text { twice } \\
\text { daily }\end{array}$ & 3 & $4,8,12$ \\
\hline & & & & propiverine (76) & $56.13 \pm 11.29$ & $20 \mathrm{mg}$ & $\begin{array}{l}\text { once } \\
\text { daily }\end{array}$ & 4 & \\
\hline \multirow[t]{2}{*}{ K.-S. Lee } & 2013 & $\mathrm{RCT}$ & Korea & imidafenacin(104) & $57.94 \pm 10.81$ & $0.1 \mathrm{mg}$ & $\begin{array}{l}\text { twice } \\
\text { daily }\end{array}$ & - & $4,8,12$ \\
\hline & & & & fesoterodine(102) & $57.63 \pm 12.63$ & $4 \mathrm{mg}$ & $\begin{array}{l}\text { once } \\
\text { daily }\end{array}$ & - & \\
\hline \multirow[t]{2}{*}{ Dmitry Y. } & 2019 & $\mathrm{RCT}$ & Russia & imidafenacin(143) & $47.2(21-66)$ & $0.2 \mathrm{mg}$ & $\begin{array}{l}\text { once } \\
\text { daily }\end{array}$ & 0 & $2,4,8,12$ \\
\hline & & & & tolterodine (146) & $46.1(19-66)$ & $4 \mathrm{mg}$ & $\begin{array}{l}\text { once } \\
\text { daily }\end{array}$ & 0 & \\
\hline
\end{tabular}

RCT, randomized controlled trial; *Withdrawal related to adverse events

\section{Figures}




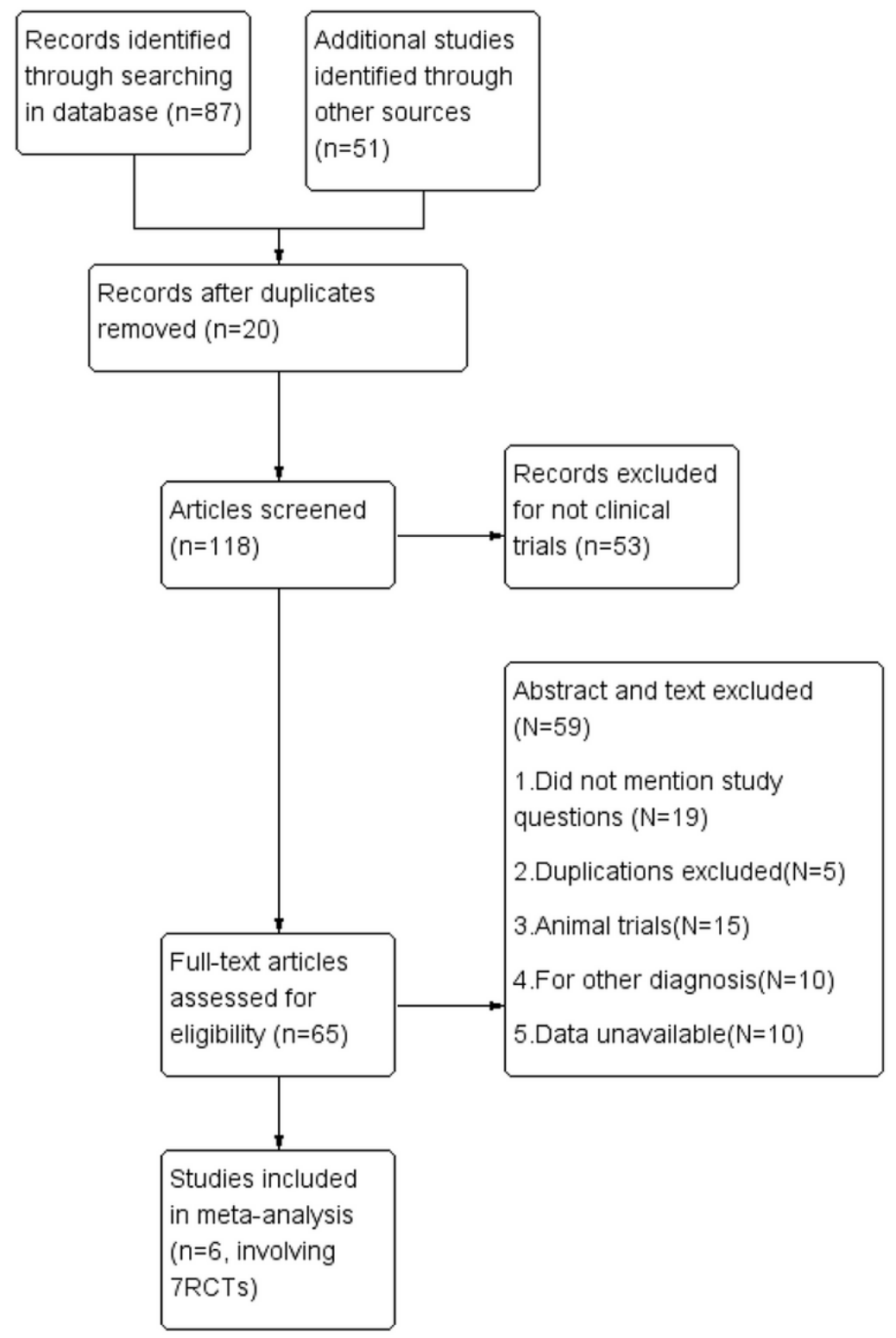

Figure 1

PRISMA flowchart of study selection. 


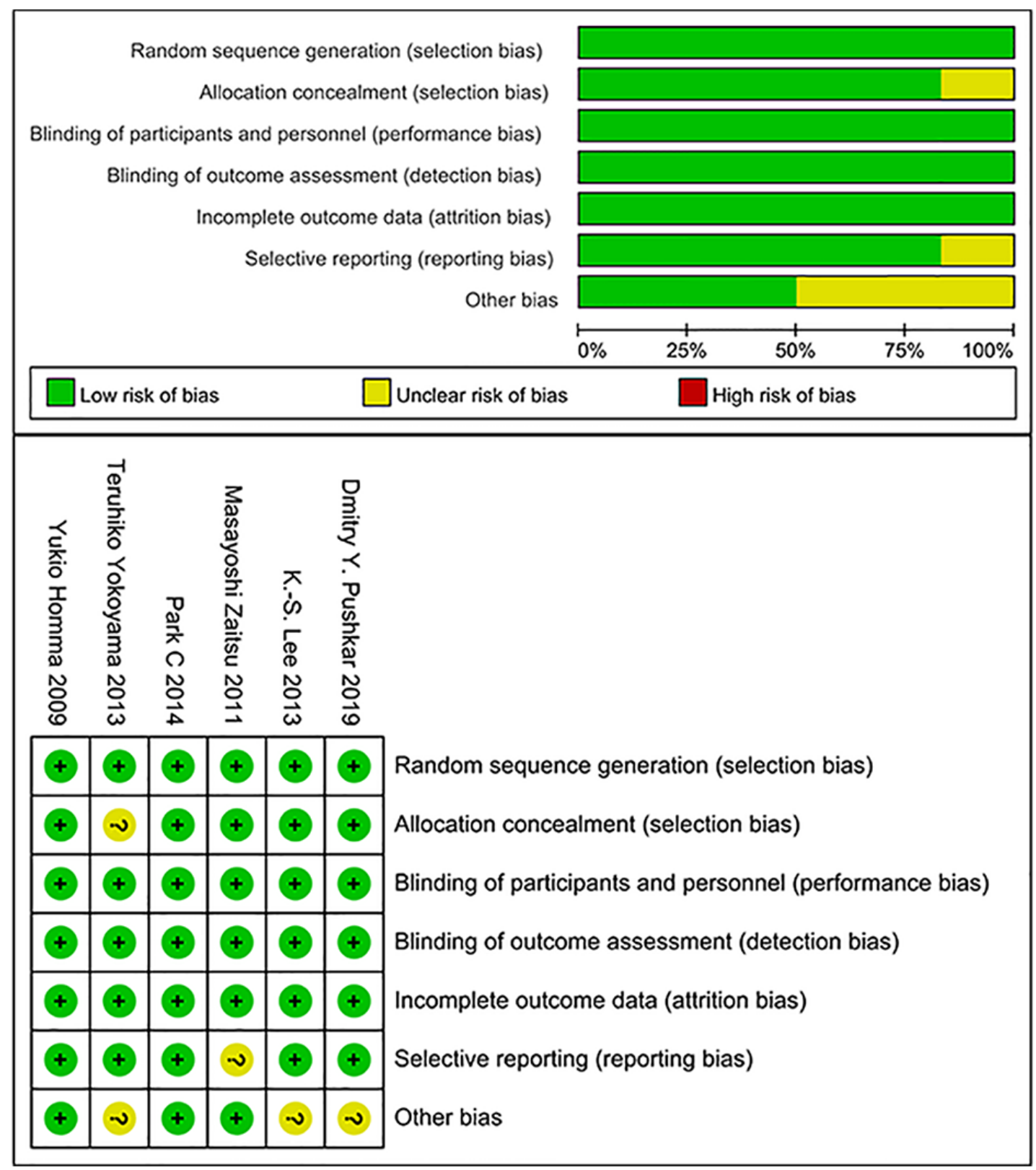

Figure 2

Results of assessment of risk of bias (ROB). ROB domains were judged as low(green), high(red), or unclear risk(yellow). 


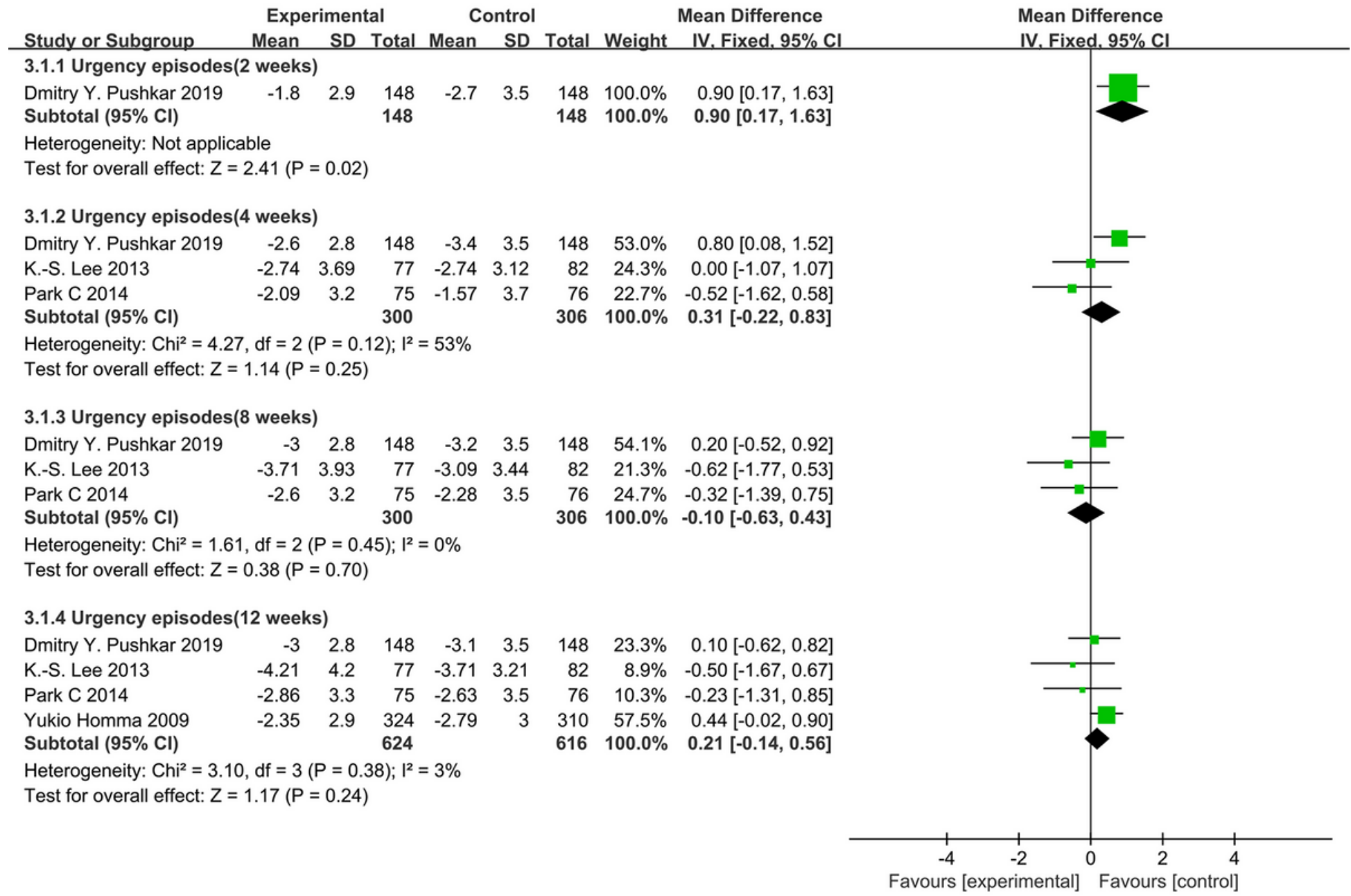

Figure 3

Forest Plot for urgency episodes per day post the treatment of imidafenacin and other antimuscarinics. 


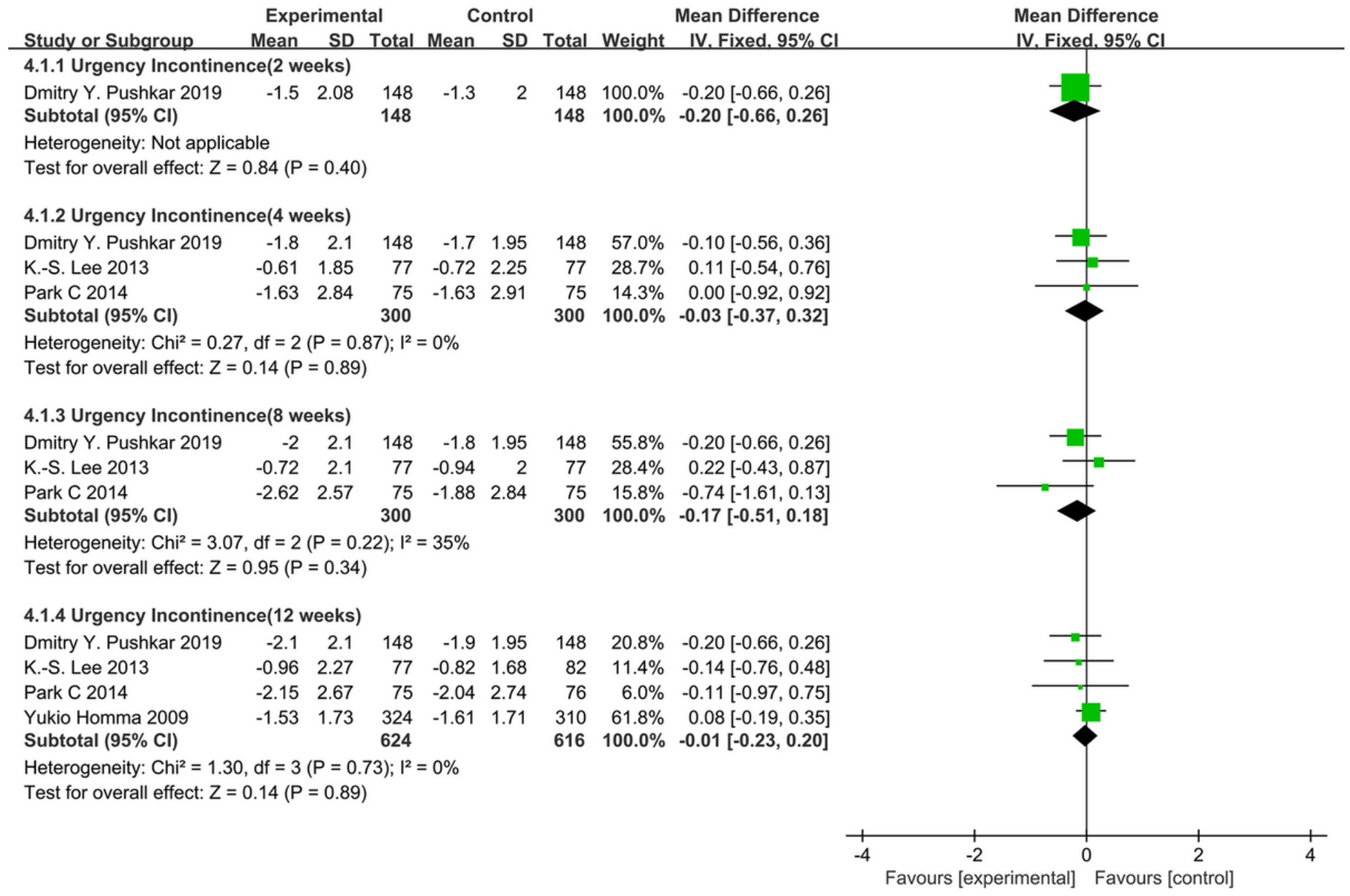

Figure 4

Forest Plot for urgency incontinence episodes per day. 


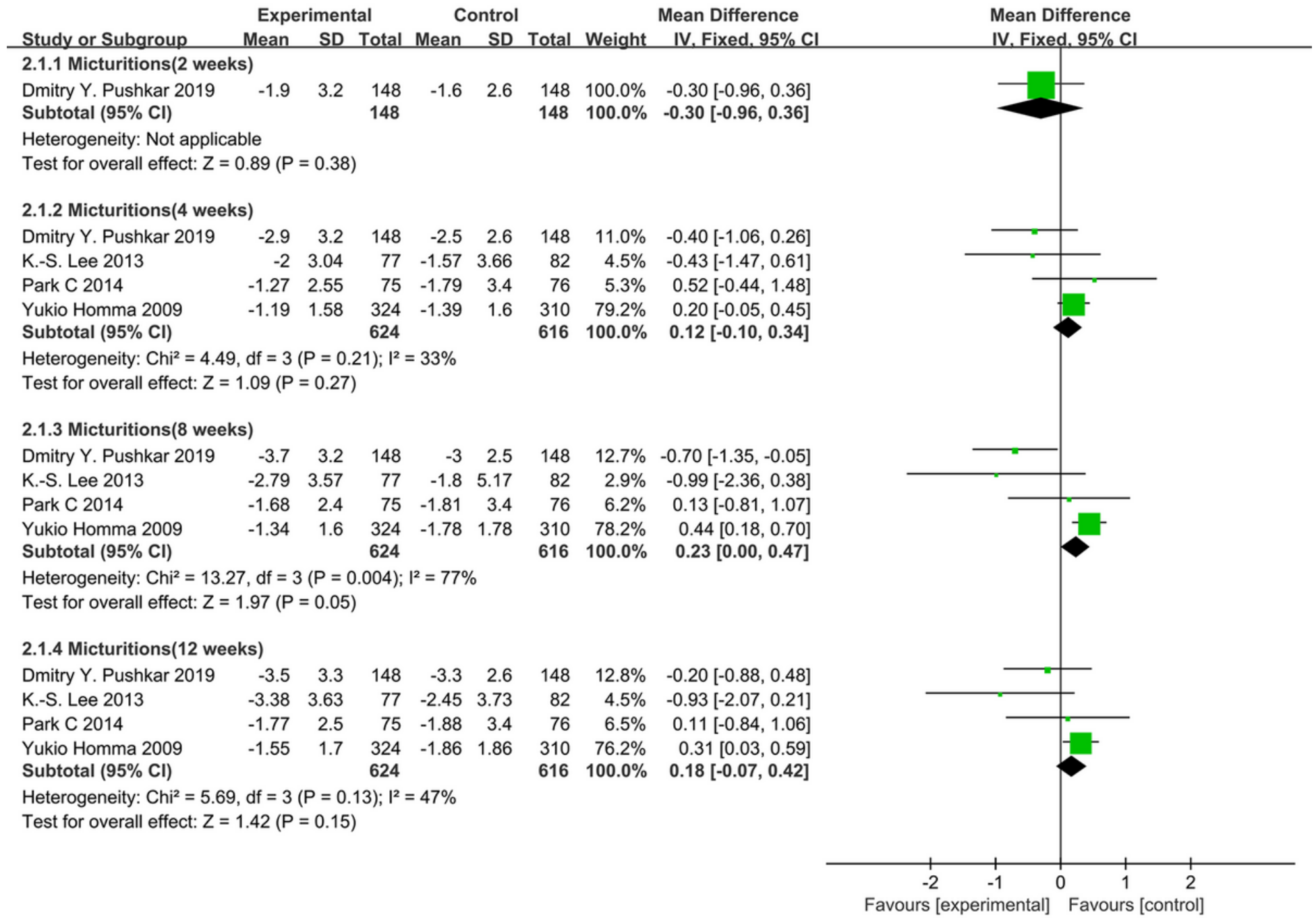

\section{Figure 5}

Forest Plot for micturition per day.

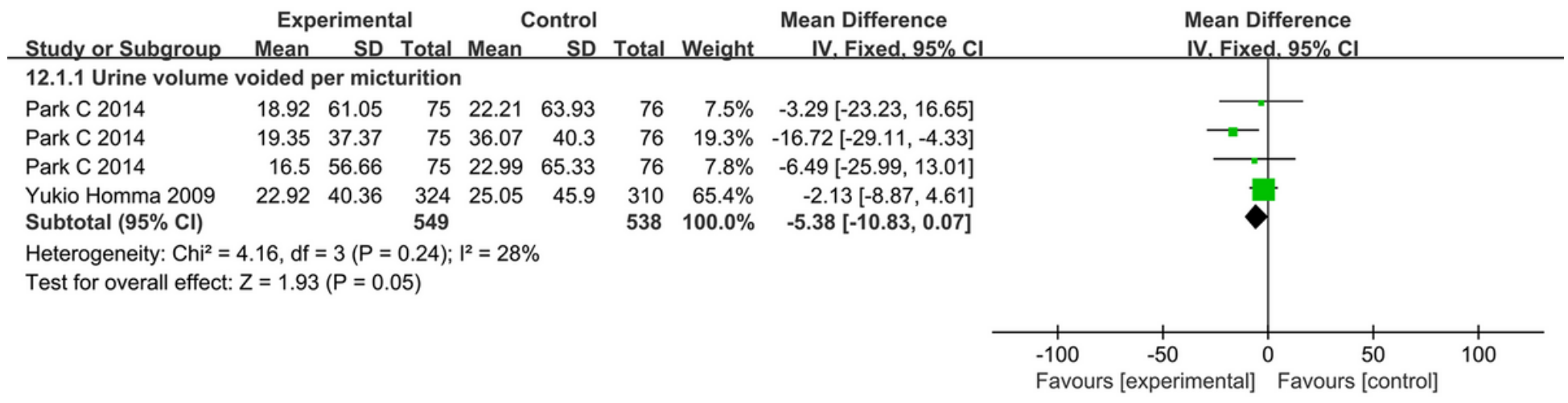

Figure 6

Forest Plot for urine volume voided per micturition. 


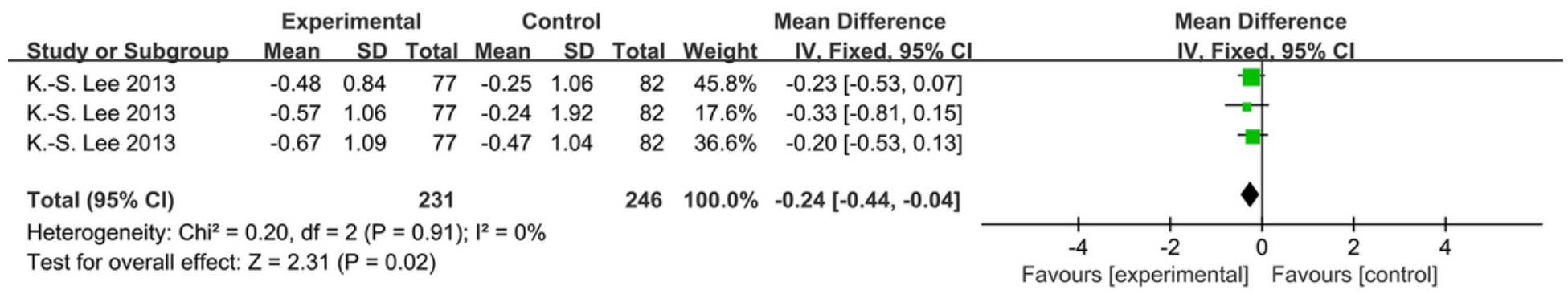

\section{Figure 7}

Forest Plot for nocturia episodes post the treatment of imidafenacin and other cholinergic drugs.

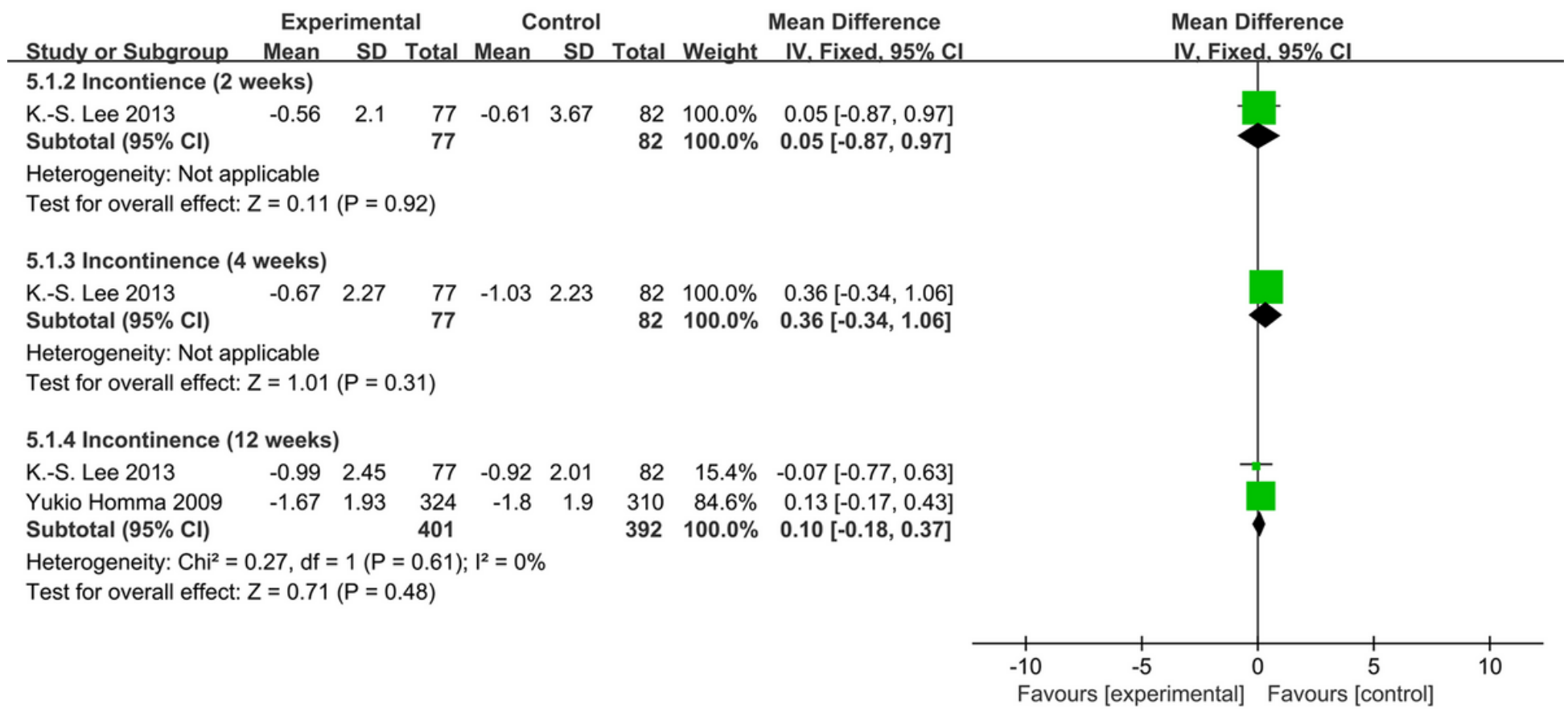

\section{Figure 8}

Forest Plot for incontinence episodes per day post the treatment of imidafenacin and controlled group. 


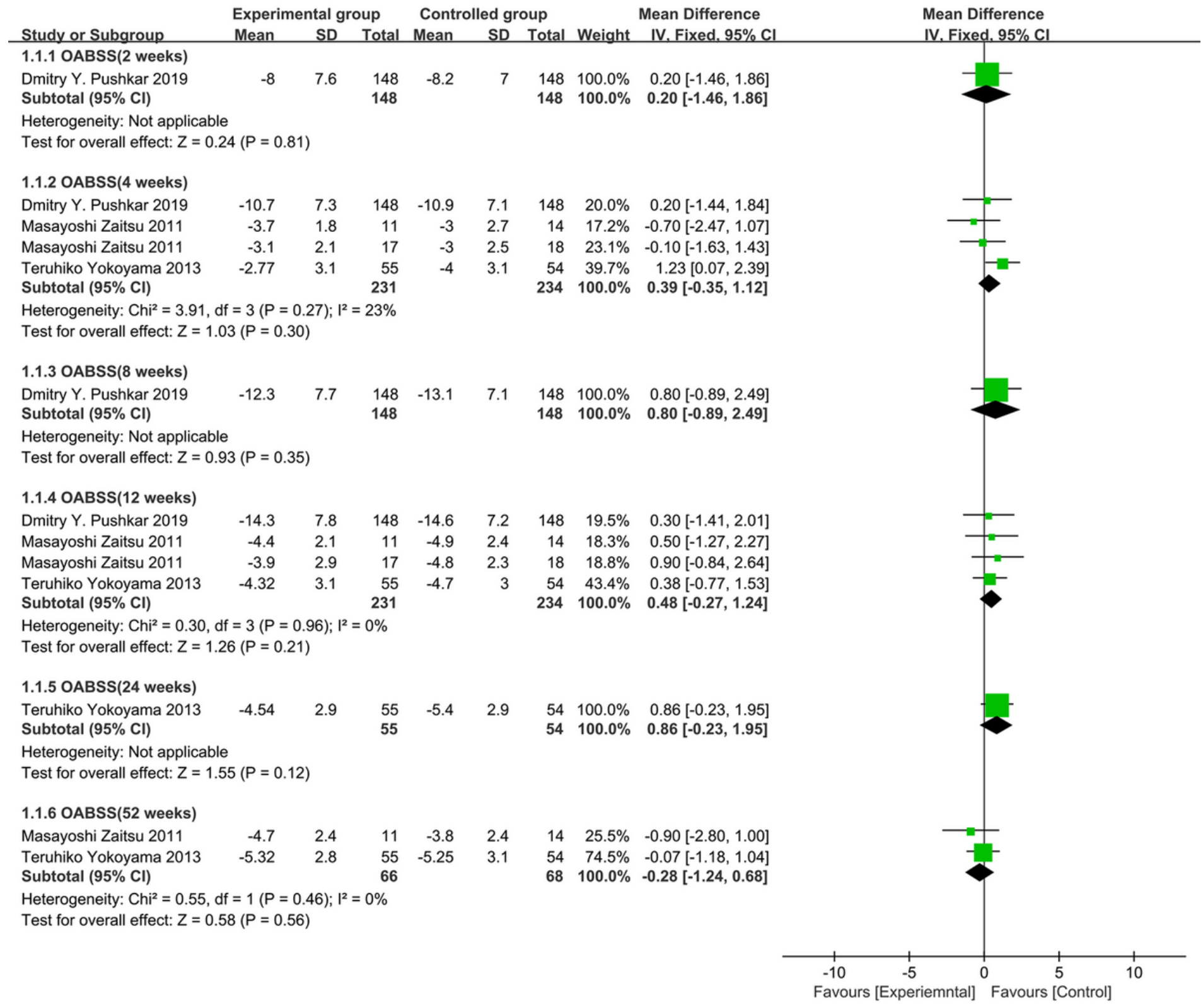

\section{Figure 9}

Forest Plot for overactive bladder symptom score (OABSS). 


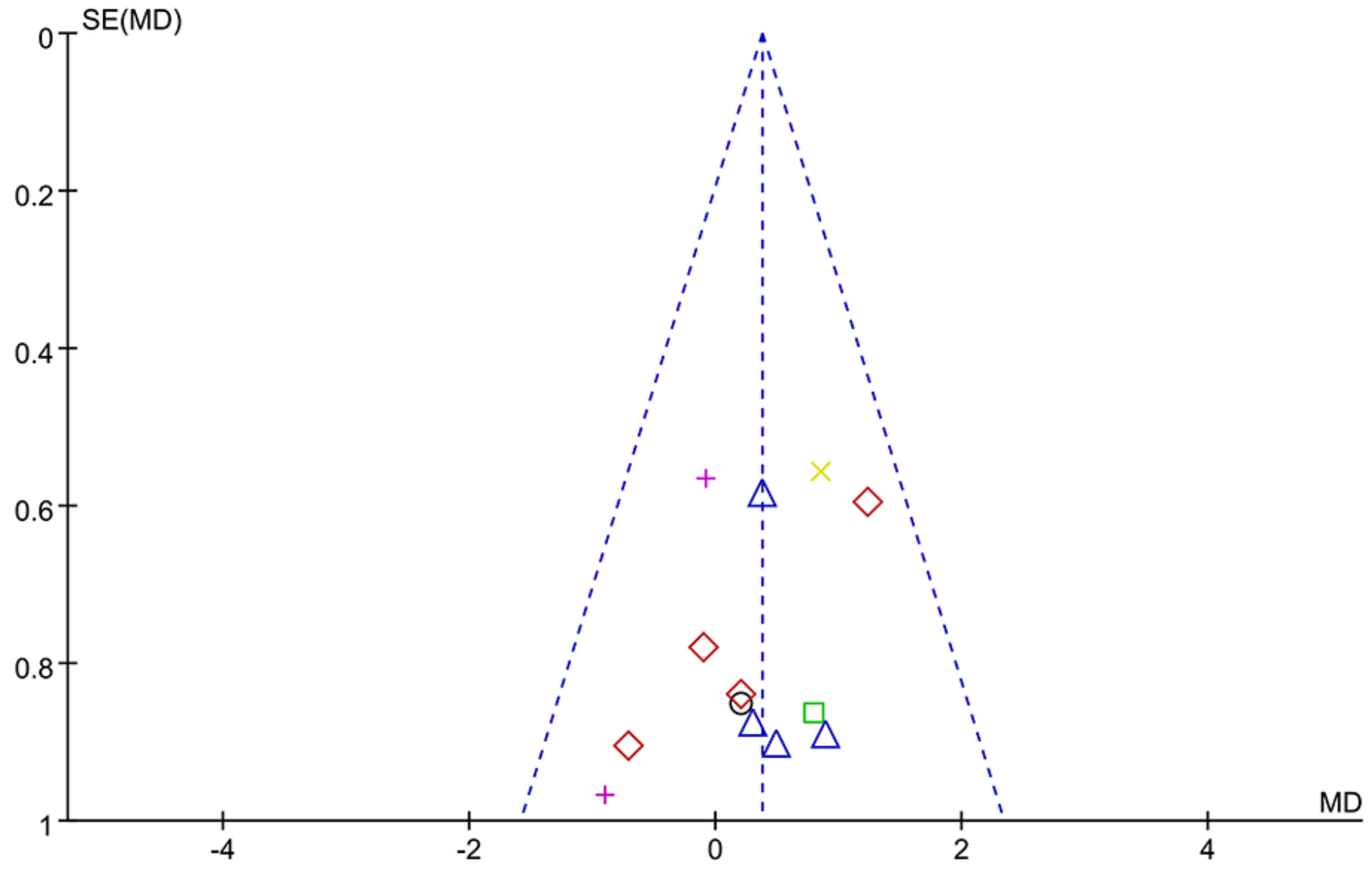

Subgroups

OABSS(2 weeks) $\square$ OABSS(8 weeks) $\quad \times$ OABSS(24 weeks)

OABSS (4 weeks) $\triangle \mathrm{OABSS}(12$ weeks) $+\mathrm{OABSS}(52$ weeks)

Figure 10

Publication bias in overactive bladder symptom score (OABSS). 


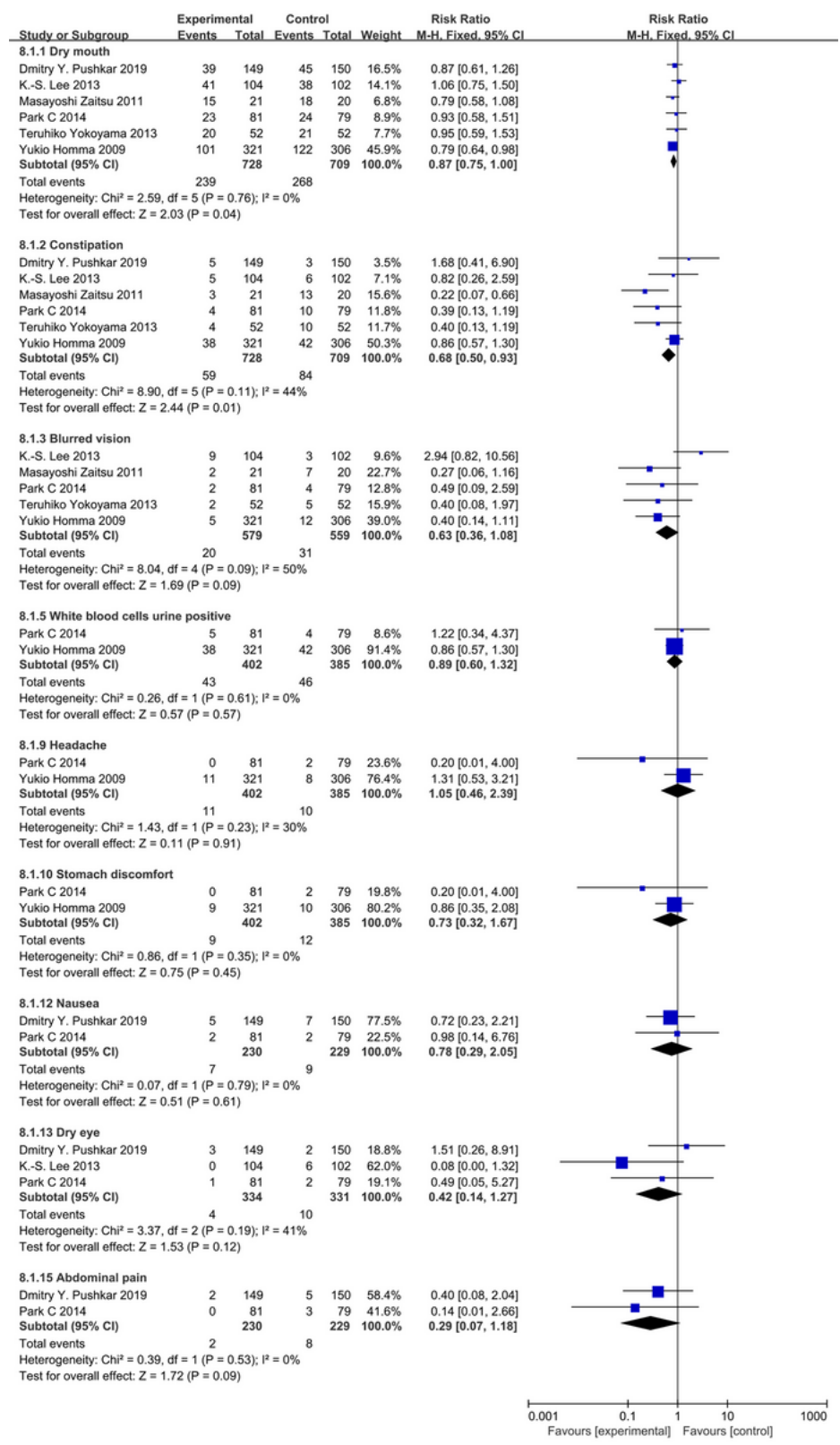

\section{Figure 11}

Forest Plot for overall adverse events post the treatment of antimuscarinics.

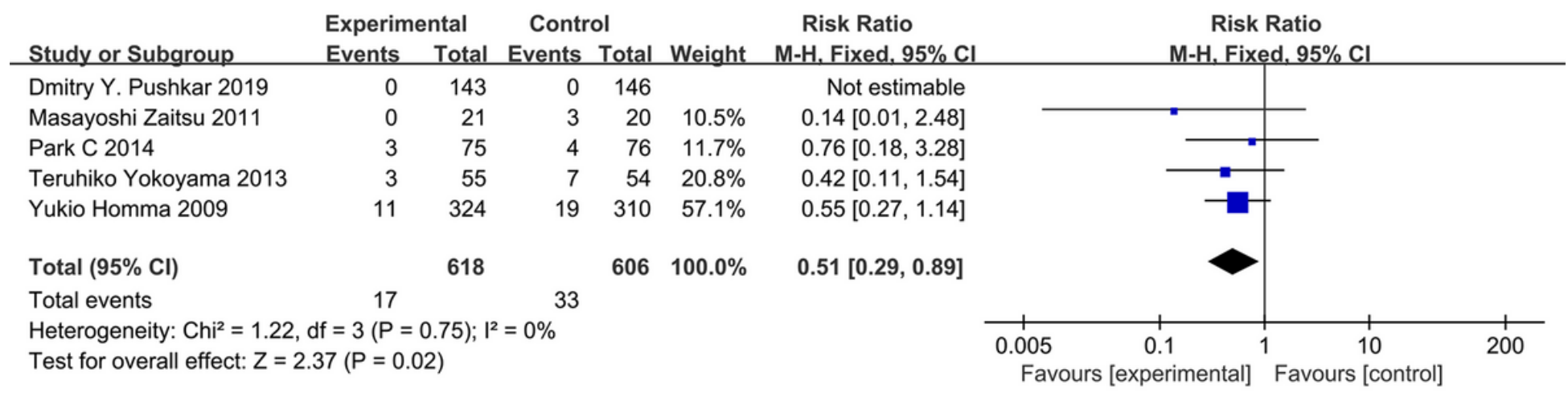

\section{Figure 12}

Forest Plot for dropout rate related to adverse events for the treatment of imidafenacin and other antimuscarinics. 


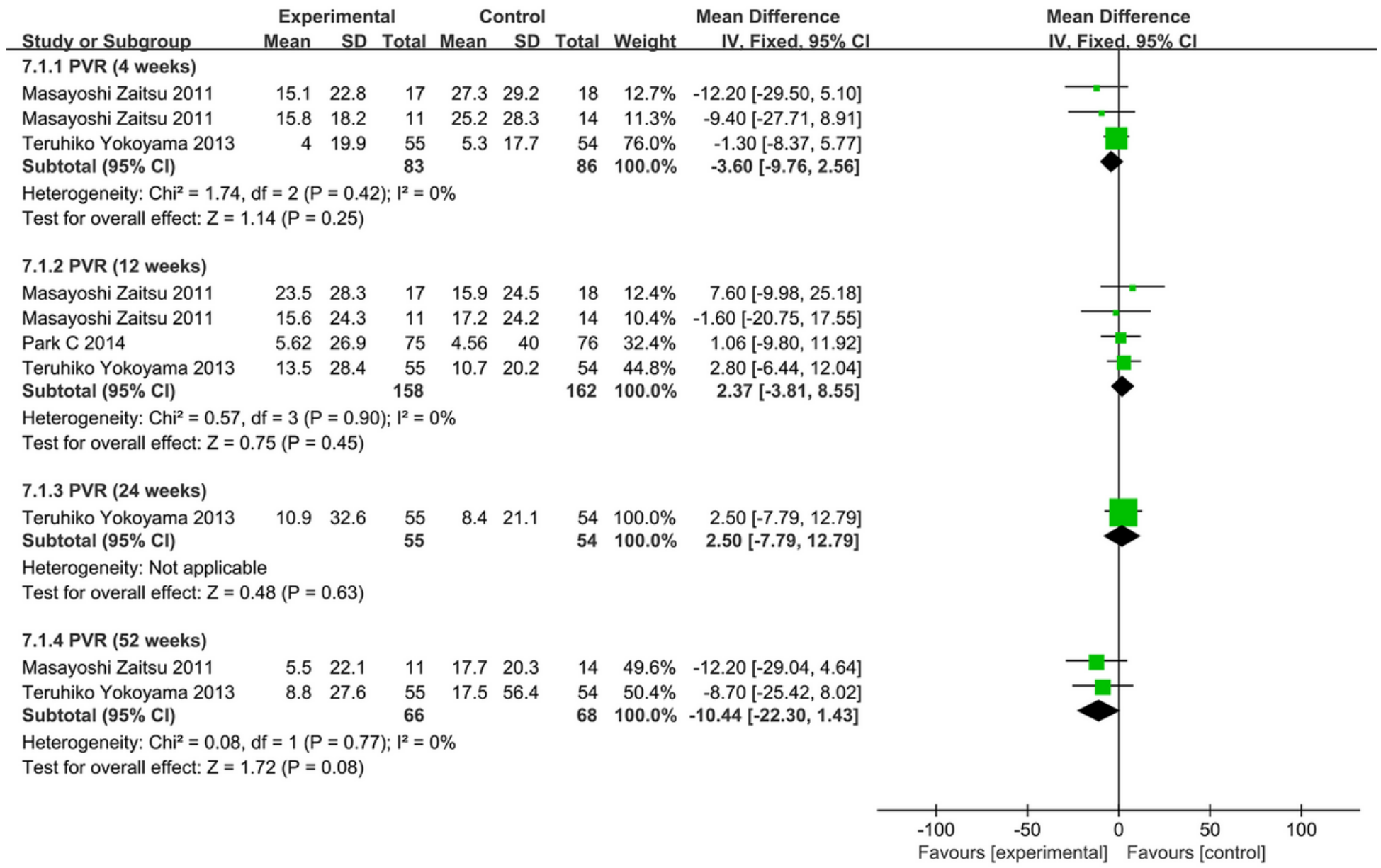

Figure 13

Forest Plot for postvoid residual volume (PVR).

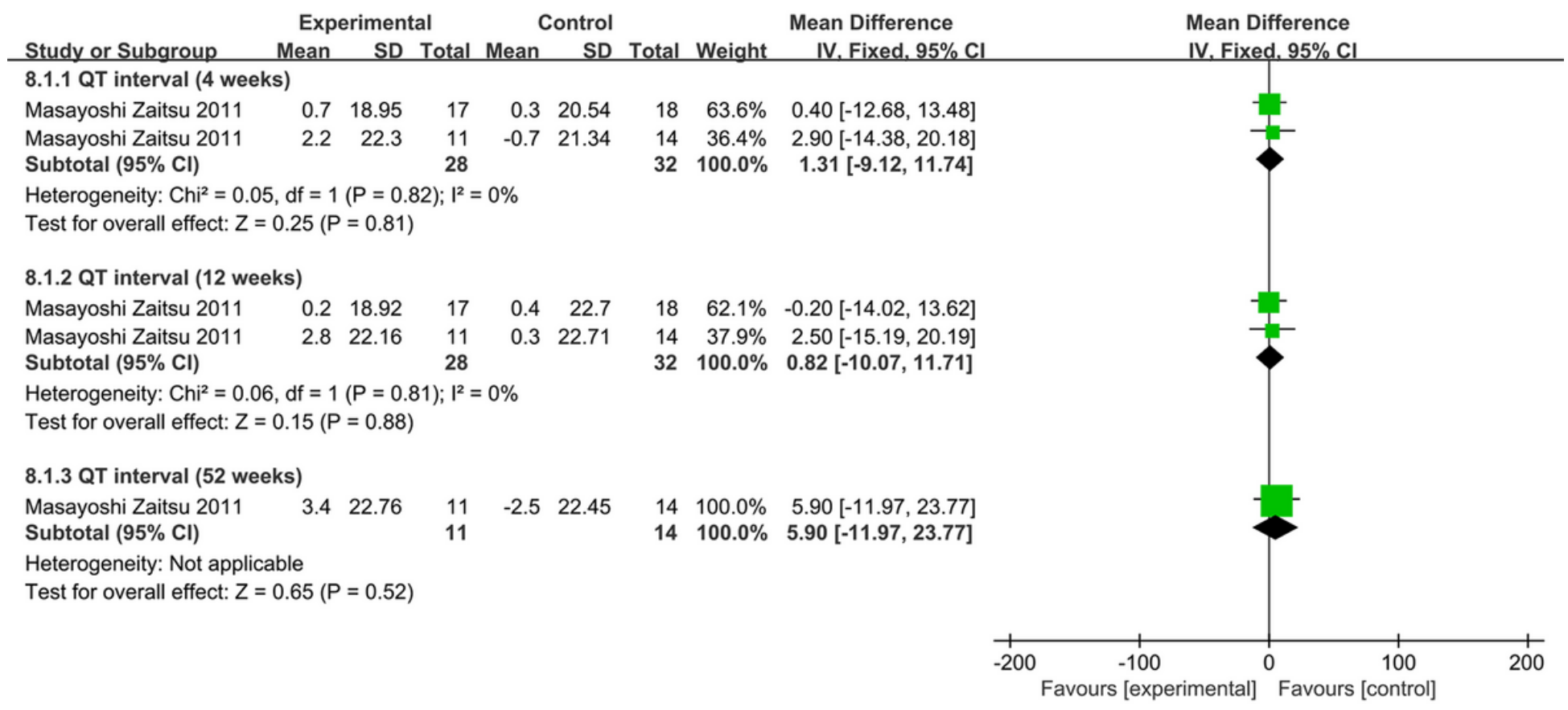

\section{Figure 14}

Forest Plot for prolongation of QT intervals of electrocardiogram (ECG). 